\title{
Comment on "Theory of the Liapunov exponents of Hamiltonian systems and a numerical study on the transition from regular to irregular classical motion"
}

\author{
Feddersen, H. K.; Flesch, R.; Salerno, M.; Christiansen, Peter Leth
}

Published in:

Journal of Chemical Physics

Link to article, DOI:

$10.1063 / 1.457997$

Publication date:

1990

Document Version

Publisher's PDF, also known as Version of record

Link back to DTU Orbit

Citation (APA):

Feddersen, H. K., Flesch, R., Salerno, M., \& Christiansen, P. L. (1990). Comment on “Theory of the Liapunov exponents of Hamiltonian systems and a numerical study on the transition from regular to irregular classical motion". Journal of Chemical Physics, 92(3), 2117-2118. https://doi.org/10.1063/1.457997

\section{General rights}

Copyright and moral rights for the publications made accessible in the public portal are retained by the authors and/or other copyright owners and it is a condition of accessing publications that users recognise and abide by the legal requirements associated with these rights.

- Users may download and print one copy of any publication from the public portal for the purpose of private study or research.

- You may not further distribute the material or use it for any profit-making activity or commercial gain

- You may freely distribute the URL identifying the publication in the public portal 


\title{
Comment on "Theory of the Liapunov exponents of Hamiltonian systems and a numerical study on the transition from regular to irregular classical motion"
}

\author{
H. K. Feddersen, R. Flesch, a) M. Salerno, b) and P. L. Christiansen \\ Laboratory of Applied Mathematical Physics, The Technical University of Denmark, Dk-2800 Lyngby, \\ Denmark
}

(Received 19 July 1989; accepted 25 October 1989)

In a recent paper ${ }^{1}$ Meyer presents a method to calculate the fraction $q$ of the phase space which is occupied by chaotic trajectories by considering only a Poincaré surface of section rather than the whole constant energy surface $H\left(x, y, p_{x}\right.$, $\left.p_{y}\right)=E$. In this comment we point out that his technique is not always applicable. In particular one must be very careful about the choice of Poincaré section as we shall illustrate in a simple example.

Meyer's results are based on the following equality which is shown in his Appendix $A^{1}$

$$
\begin{aligned}
\langle f\rangle_{s} & \equiv \int_{S_{E}} d x d p_{x} f(\tilde{\gamma})=\int_{\Gamma} d \gamma \nu(\gamma) \delta[H(\gamma)-E] f(\gamma) \\
& \equiv\langle v f\rangle_{\Gamma}
\end{aligned}
$$

where $\gamma=\left(x, y, p_{x}, p_{y}\right)$ is a point in the phase space $\Gamma, H$ is the Hamiltonian, $\tilde{\gamma}=\tilde{\gamma}\left(x, p_{x}\right)$ is a point in the Poincare surface of section $S_{E}, v(\gamma)$ denotes the average frequency with which the trajectory started at the phase space point $\gamma$ intersects the Poincare section, and $f$ is some constant of motion.

Choosing $f$ to be the constant of motion, $X(\gamma)$, where

$X(\gamma)= \begin{cases}0 & \text { if } \gamma \text { is a point on a regular trajectory } \\ 1 & \text { if } \gamma \text { is a point on a chaotic trajectory }\end{cases}$

we have for the fraction $q$ of chaotic phase space ${ }^{1}$

$$
q=\frac{\int_{\Gamma} d \gamma X(\gamma) \delta[H(\gamma)-E]}{\int_{\Gamma} d \gamma \delta[H(\gamma)-E]}=\frac{\langle X\rangle_{\Gamma}}{\langle 1\rangle_{\Gamma}} .
$$

This definition requires us to examine integrals of the form

$$
\langle f\rangle_{\Gamma}=\int_{\Gamma} d \gamma \delta[H(\gamma)-E] f(\gamma)
$$

To evaluate this integral Meyer "inverts" (1) obtaining $\langle f\rangle_{\Gamma}=\left\langle v^{-1} f\right\rangle_{s}$. However, this is in general not true, that is we are not able to obtain information about the whole energy surface by considering only a Poincaré section. Below we show that the equation $\langle f\rangle_{\Gamma}=\left\langle v^{-1} f\right\rangle_{s}$ holds only if we use a Poincare section $S_{E}$ that is intersected by all of the trajectories on the energy surface. In general such a surface will be difficult to find if it exists at all.

To prove the above assertion we assume that there exist trajectories which do not intersect the Poincare section. We denote by $\Gamma_{1}$ the subset of the energy surface consisting of all points on the trajectories which do intersect the Poincare section, that is

$$
\Gamma_{1}=\{\gamma \in \Gamma \mid v(\gamma) \neq 0\}
$$

since $v(\gamma)=0$ for $\gamma \notin \Gamma_{1}$ we have

$$
\begin{aligned}
& \int_{\Gamma_{1}} d \gamma v(\gamma) \delta[H(\gamma)-E] f(\gamma) \\
&=\int_{\Gamma} d \gamma v(\gamma) \delta[H(\gamma)-E] f(\gamma)=\langle f\rangle_{s}
\end{aligned}
$$

and from the definition of $\Gamma_{1}$

$$
\begin{aligned}
\left\langle v^{-1} f\right\rangle_{s} & =\int_{S_{E}} d x d p_{x} v^{-1}(\tilde{\gamma}) f(\tilde{\gamma}) \\
& =\int_{\Gamma_{1}} d \gamma \delta[H(\gamma)-E \mathrm{l} f(\gamma) .
\end{aligned}
$$

By our assumption $\Gamma_{1}$ is not the entire energy surface, hence

$$
\int_{\Gamma} d \gamma[H(\gamma)-E] f(\gamma) \neq \int_{\Gamma_{1}} d \gamma \delta[H(\gamma)-E] f(\gamma)
$$

and therefore

$$
\langle f\rangle_{r} \neq\left\langle v^{-1} f\right\rangle_{s} .
$$

In the special case in which all of the trajectories do intersect the Poincare section, $\Gamma_{1}$ is identical to the entire energy surface and Meyer's result follows. We note that in the examples treated in Ref. 1 this is in fact the case.

For example, consider two uncoupled harmonic oscillators given by the Hamiltonian

$$
H=x^{2}+y^{2}+p_{x}^{2}+p_{y}^{2},
$$

where the energy is taken to be $H=1$.

Since the general solution is sinusoidal with period $2 \pi$ we have

$$
v(\gamma)=1 / 2 \pi
$$

Now, define the Poincare surface of section as

$$
S_{E}=\left\{\left(x, p_{x}\right) \mid \exists p_{y}>0: \quad H\left(x, y=c, p_{x}, p_{y}\right)=1\right\},
$$

where $c$ is a constant, $|c|<1$. The set of $\left(x, p_{x}\right)$ lying in $S_{E}$ is then given by

$$
p_{y}^{2}=1-x^{2}-y^{2}-p_{x}^{2}=1-x^{2}-c^{2}-p_{x}^{2}>0
$$

or

$$
x^{2}+p_{x}^{2}<1-c^{2}
$$

In the following we let $f(\gamma)=1$ and compare $\langle f\rangle_{\Gamma}$ and $\left\langle v^{-1} f\right\rangle_{s}$ for different Poincare sections, that is for different values of $c$. The integral $\left\langle v^{-1} f\right\rangle_{s}=\left\langle v^{-1}\right\rangle_{s}$ over the Poincaré section is thus 


$$
\begin{aligned}
\left\langle v^{-1}\right\rangle_{s} & =\int_{S_{E}} d x d p_{x} v^{-1}(\gamma)=2 \pi \int_{S_{E}} d x d p_{x} \\
& =2 \pi \cdot \pi\left(1-c^{2}\right)=2 \pi^{2}\left(1-c^{2}\right) .
\end{aligned}
$$

The integral $\langle 1\rangle_{\Gamma}$ over the whole energy surface is ${ }^{2}$

$$
\begin{aligned}
\langle 1\rangle_{\Gamma} & =\int d \gamma \delta[H(\gamma)-1] \\
& =\text { surface volume of a four dimensional unit sphere } \\
& =2 \pi^{2} .
\end{aligned}
$$

Hence $\left\langle v^{-1}\right\rangle_{s}=\langle 1\rangle_{\Gamma}$ only when the Poincaré section is given by $y=c=0$, that is when all trajectories of the entire energy surface intersect the actual Poincaré section.

This example illustrates how important it is to choose the correct Poincare section. One should also note that such a section which is intersected by all trajectories on the energy surface might not exist for other Hamiltonians in which case Meyer's method will never work. Therefore a different method for evaluation of $q$ must be applied. ${ }^{3}$

The financial support of the Danish Research Council for scientific and Industrial research and Thomas B. Thriges Fond is acknowledged by R. F. and M. S.

\footnotetext{
a) Current address: Heriot-Watt University, Department of Mathematics, Riccarton, Edinburgh EH14 4AS, Scotland.

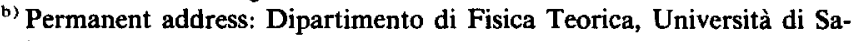
lerno, 84100 Salerno, Italy.

'H.-D. Meyer, J. Chem. Phys. 84, 3147 (1986).

${ }^{2}$ H. Flanders, Differential Forms with Applications to the Physical Sciences (Academic, New York, 1963), Chapter 6.

${ }^{3}$ L. Cruzeiro-Hansson, H. Feddersen, R. Flesch, P. L. Christiansen, M. Salerno, and A. C. Scott (submitted).
}

\title{
Does squaring the quantum Monte Carlo weights give the exact quantum probability distribution?
}

\author{
Peter J. Reynolds \\ Physics Division, Office of Naval Research, 800 North Quincy Street, Arlington, Virginia 22217
}

(Received 26 January 1989; accepted 23 October 1989)

There is considerable current interest in the use of quantum Monte Carlo methods (or Green's function Monte Carlo) for the accurate determination of energies and energy-related quantities in systems both large and small. ${ }^{1}$ Such systems range from liquid helium and metallic hydrogen, down to atoms, molecules, and clusters. This interest arises because quantum Monte Carlo ( $\mathrm{QMC}$ ) can in principle provide an exact, fully correlated, stochastic (i.e., random walk) solution to the Schrödinger equation. ${ }^{1,2}$ Unfortunate$l y$, it is far more difficult to use QMC to obtain expectation values of quantities unrelated to the energy, such as dipole and quadrupole moments, mean lengths (e.g., mean interelectronic separations of like- or unlike-spins and their moments), and any other operators which do not commute with the Hamiltonian. Evaluation of these quantities typically requires the use of auxilliary Monte Carlo calculations ${ }^{3-5}$ that are costly (in computer time), and increase the statistical uncertainty with which the expectation values are ultimately ascertained.

Recently, East et al. ${ }^{6}$ have published a QMC approach which does not require auxiliary walks. Their method appears both simple and elegant. Unfortunately, the authors have overlooked a subtle point involving the order in which averaging and squaring are performed. Below we follow their logic to reveal the error, and to obtain the correct expression for the weight and its square. First, however, we demonstrate that their approach does not lead to the correct asymptotic probability distribution by considering their algorithm and the Green's function which describes it. From the Green's function we obtain the Schrödinger-like equa- tion for the probability density. The exact density $|\phi|^{2}$ is not a solution to this equation, in contrast to the requirement of their claim.

In the short-time approximation to QMC (generally known as diffusion QMC), an approximation to the Green's function for the following differential equation is iterated to large time and steady state ${ }^{2}$ :

$$
\frac{\partial f}{\partial t}=D \nabla^{2} f+\left[E_{0}-E_{L}\right] f-D \nabla \cdot(f \mathbf{F}) .
$$

Equation (1) is derived through an importance-sampling transformation of the Schrödinger equation. ${ }^{2,7}$ The probability density $f=\Psi_{T} \phi$ is a product of a known trial wave function $\Psi_{T}(\mathbf{R})$, and an unknown function $\phi(R, t)$ which in the steady-state (at large time) is the exact, unknown state of interest (generally the ground state). The functions $\mathbf{F}$ and $E_{L}$ are simply related to $\Psi_{T}$, while $E_{0}$ is a constant, representing a shift of the zero of energy (also generally to the ground state). A distribution of points chosen from $f$ is obtained by iterating the Green's function solution of Eq. (1).

$$
\begin{aligned}
& \text { Writing } \\
& \widetilde{H}=\widetilde{V}+\widetilde{T},
\end{aligned}
$$

where

$$
\widetilde{V}=E_{L}-E_{0}
$$

and

$$
\widetilde{T}=-D \nabla^{2}+D \nabla \cdot \mathbf{F}+D \mathbf{F} \cdot \nabla
$$

we may rewrite Eq. 1 as 